\title{
On the acoustics of a coupled space
}

\author{
Charles Thompson \\ Department of Engineering Science and Mechanics, Virginia Polytechnic Institute and State University, \\ Blacksburg, Virginia 24061
}

(Received 2 March 1983; accepted for publication 2 October 1983)

\begin{abstract}
An examination of acoustic wave propagation in a coupled space is presented. The analysis presented is limited to the first two longitudinal modes of the cavity. It is shown that the spacial behavior of the modes of vibration in the cavity is affected by the coupling discontinuity. The degree with which the discontinuity influences the pressure variation is parametrized by a single small parameter $\epsilon$, where $\epsilon$ is the ratio of the typical cavity height, $H_{0}$ to the cavity length $L_{0}$. An approximate solution for the pressure in the space is obtained using the method of matched asymptotic expansions. Experimental results are also presented as verification of the theoretical results.
\end{abstract}

PACS numbers: 43.20.Ks, 43.20.Bi, 43.55.Br

\section{INTRODUCTION}

The propagation of an acoustic pressure wave in a coupled space has long been of interest to auditorium acousticians. Davis ${ }^{1}$ and Eyring ${ }^{2}$ have derived expressions for determining the reverberation time of rooms coupled through an adjoining wall. These theories are based on the assumption that the sound field is diffuse in each of the rooms and the absorption coefficient of the walls is uniform. However, in situations involving low-frequency and/or nonuniform wall absorption an acoustic analysis using statistical theory is no longer valid. Hence, the boundary value problem must be solved. For this reason Bolt ${ }^{3}$ and Harris and Feshbach worked toward determining the effect of boundary perturbations on the propagation of acoustic waves in a coupled space. Bolt, for a similar geometry to that given in Fig. 1, showed experimentally that the isobars of the first two longitudinal modes of the cavity were nonplanar near the junction, but gave no explanation for this result. Harris and Feshbach ${ }^{4}$ examined both experimentally and analytically

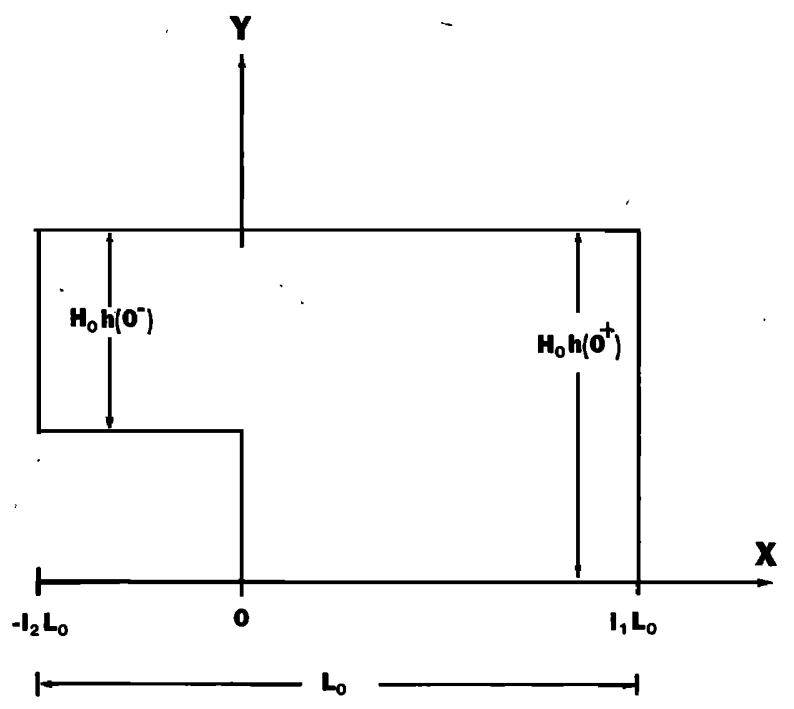

FIG. 1. Plan view of a coupled space. how a planar coupling aperture effects the resonant frequencies of an enclosure. However, they made no mention as to the degree with which the aperture effected the spatial variation of the pressure in the enclosure. Kagawa and Omote used a finite-element method to examine plane-wave propagation in acoustic filters of circular cross section. In the experimental phase of their work they found that at a wall discontinuity the wave front was spherical in shape. As they moved away from the discontinuity in the axial direction, the wave front became planar. They attributed the discrepancies between the calculated and measured pressure near the discontinuity to inadequate sampling of the space. Lesser and Lewis ${ }^{6}$ determined the acoustic impedance across a step discontinuity in an infinite two-dimensional waveguide with slowly varying height using the method of matched asymptotic expansions. However, they neglected to incorporate transcendentally small terms into the asymptotic representation of the pressure near the discontinuity. For a waveguide having infinite length these terms can be justifiably dropped. However, in a cavity these terms determine the critical distance between the wall and the discontinuity at which interaction between the incompressible and wave regions take place. Thompson ${ }^{7,8}$ has obtained uniform solution for the pressure behavior for a waveguide which has a single boundary discontinuity. Using an analytical development similar to that given by Thompson ${ }^{7}$ we will show that the nonplanar behavior of the pressure near the junction of the two cavities coupled by a step discontinuity is the result of incompressible fluid motion near the junction.

In this paper a uniformly valid asymptotic representation of the standing wave pattern will be determined using. the method of matched asymptotic expansions, MMAE. We will split the cavity into three regions. In the regions of space, between $X$ equal to $-l_{2} L_{0}$ and $0^{-}$and $0^{+}$and $l_{1} L_{0}$ the length scale which parametrizes the fluid's motion is the acoustic wavelength. Hence they will be called wave regions. In the region of space between $X$ equal to $0^{-}$and $0^{+}$the length scale which parametrizes the fluid's motion is $H_{0}$. Since $H_{0}$ is small compared to an acoustic wavelength this region will be termed incompressible. The frequency will be 
held to the range where only longitudinal modes in $X$ are excited. Despite this frequency limitation sufficient insight can be gained into the difficulties we would encounter if we were to embark on a straightforward numerical attack of the problem, i.e., under sampling the space. The perturbation parameter $\epsilon$ in the coupled space problem is taken to equal the ratio of the maximum cavity height to the total cavity length. The parameter $\epsilon$ is assumed to be much less than one (see Fig. 1).

Sections I and II will be devoted to the nondimensionalization of the linear inviscid equations which govern the wave and incompressible regions, respectively. The pressure and particle velocity in each region will be represented in terms of a truncated asymptotic sequence in $\epsilon$. In Sec. III the composite solution will be presented. In Sec. IV we will compare and discuss the theoretical and experimental results of our analysis.

\section{THE WAVE REGION}

In the range frequencies where $\omega H_{0} / c<1$ we expect the pressure, away from the discontinuity, to be uniform in $Y$. Near resonance the acoustic wavelength $\lambda$ is of $O\left(L_{0}\right)$. Using this information the fluid's behavior in the cavity can be parametrized by the single small parameter $\epsilon$, which equals $H_{0} / L_{0} . H_{0}$ is taken to be the maximum height of the cavity and $L_{0}$ the cavity length.

The nondimensionalization of the horizontal and vertical velocities as well as the pressure is as follows:

$$
u=U / U_{0}, \quad v=V /\left(U_{0} \epsilon\right),
$$

and

$$
p=P /\left(\omega \rho_{0} L_{0} U_{0}\right) .
$$

For the coordinates the nondimensionalization is

$$
x=X / L_{0}
$$

and

$$
y=Y / H_{0},
$$

where

$$
t=\omega T, \quad k=\omega L_{0} / c,
$$

and

$$
\epsilon=H_{0} / L_{0}
$$

The nondimensional linear inviscid equations of motion in the wave region are

$$
\dot{u}+p_{x}=0, \quad \epsilon^{2} \dot{v}+p_{y}=0,
$$

and

$$
u_{x}+v_{y}+k^{2} \dot{p}=0,
$$

with the boundary conditions

and

$$
u=0 \text { at } x=-l_{2}, \quad x=l_{1},
$$

$$
v=0 \text { at } y=0, y=h^{+} \text {, and } h^{-} \text {. }
$$

As we can see from the aforementioned equations, at zeroth order in $\epsilon$ the zero normal velocity boundary condition cannot be satisfied when $x$ equals zero. Hence these equations are singular in the limit of $x$ approaching zero. However these equations accurately describe the fluid motion outside the region of influence of the discontinuity. The regions space where Eq. (1) is valid will be termed wave regions.

For our purpose it is sufficient to assume that all state variables in Eq. (1) have a harmonic time dependence of $e^{-i t}$. Under this assumption $u, v$, and $p$ represent complex amplitudes.

We will express the complex amplitudes, $p, u$, and $v$ in terms of an asymptotic sequence in integer powers of $\epsilon$. Successive approximations for the pressure and particle velocities can be determined by evaluating higher order coefficients of this sequence.

$$
(p, u, v)=E_{2}(p, u, v)
$$

where

$$
E_{2} f=f_{0}+\epsilon f_{1}+\epsilon^{2} f_{2}+O\left(\epsilon^{3}\right) .
$$

In the wave region the coefficients of the asymptotic sequence for the pressure satisfy the homogeneous one-dimensional Helmholtz equation to $O\left(\epsilon^{3}\right)$.

$$
\left(\frac{d^{2}}{d x^{2}}+k^{2}\right)\left(p_{0}+\epsilon p_{1}+\epsilon^{2} p_{2}\right)=O\left(\epsilon^{3}\right) .
$$

The solution of the one-dimensional Helmholtz equation at each order of approximation is simply the sum of two plane waves.

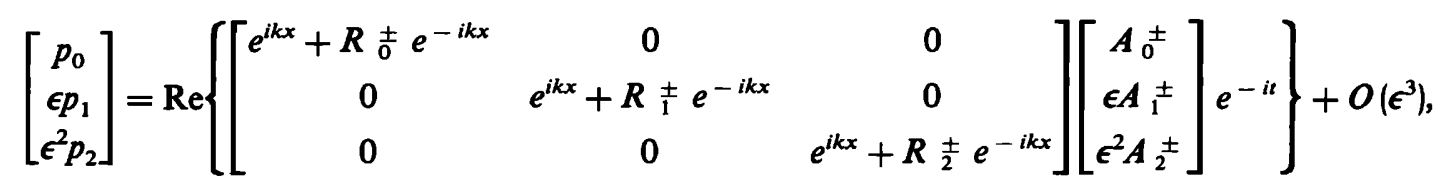

where the superscript denotes on which side of the discontinuity we are tabulating the pressure. The pressure in the wave region must satisfy the boundary conditions

$$
E_{2} p^{\prime}=0 \text { at } x=l_{1}
$$

and

$$
E_{2} p^{\prime}=0 \text { at } x=-l_{2} \text {. }
$$

\section{THE INCOMPRESSIBLE REGION}

Now let us direct our attention to the motion of the fluid in the region near $x$ equal to zero. As $x$ approaches $0^{+}$and $0^{-}$the vertical particle velocity $v$ must become of $O(1 / \epsilon)$ if we are to satisfy the zero normal velocity boundary condition on the surface of the discontinuity. Hence a locally valid set of equations must be derived for the region of space near 
the discontinuity. Following similar arguments to those given in Ref. 7 we will use the following nondimensionalization scheme in the incompressible region:

$$
\begin{array}{ll}
\hat{u}=u, & \hat{v}=v \epsilon, \\
\hat{p}=p, & \hat{x}=x / \epsilon,
\end{array}
$$

and

$$
\hat{\boldsymbol{y}}=\boldsymbol{y} .
$$

The equations governing the fluid motion in the incompressible region are

$$
\epsilon \dot{\hat{u}}+\hat{p}_{\hat{x}}=0, \quad \epsilon \dot{\hat{v}}+\hat{p}_{\hat{y}}=0,
$$

and

$$
\hat{u}_{\hat{x}}+\hat{v}_{\hat{\jmath}}=-k^{2} \dot{\hat{p}},
$$

with boundary conditions

$$
(\hat{u}, \hat{v}) \cdot \mathbf{n}=\mathbf{0},
$$

where $\mathbf{n}$ is the outward pointing normal vector to the boundary.

The coefficients for the asymptotic sequence for pressure in the incompressible region are governed by the Laplace equation at $O(\epsilon)$ and the Poisson equation at $O\left(\epsilon^{2}\right)$. From Eq. (5)

$$
\begin{aligned}
& \hat{p}_{1_{x g}}+\hat{p}_{1_{g g}}=0, \\
& \hat{p}_{2_{\ell x}}+\hat{p}_{2_{g g}}=-k^{2} \hat{p}_{0} .
\end{aligned}
$$

The zeroth order pressure coefficients are

$$
\hat{p}_{0}=\text { const }=p_{0}\left(0^{+}\right)=p_{0}\left(0^{-}\right) .
$$

If we let

$$
\hat{p}_{2}=\hat{\mathbf{p}}_{2}-k^{2} \hat{p}_{0} \hat{x}^{2} / 2 \text {, }
$$

we can rewrite Eq. (6) as a set of two homogeneous Laplace equations

$$
\begin{aligned}
& \hat{p}_{1_{x x}}+\hat{p}_{1_{g g}}=0, \\
& \hat{\mathbf{p}}_{2_{g x}}+\hat{\mathbf{p}}_{2_{g g}}=0,
\end{aligned}
$$

with boundary conditions that the normal velocity equals zero on the surface of the step discontinuity. The solution of the Laplace equation for the pressure in the coupled enclosure which has a step discontinuity in height will be solved using the Schwartz Christoffel transformation.

The conformal transform from the physical plane, $\tilde{z}$ plane, to the upper half $\omega$ plane, can be made using the relation

$$
\frac{d \tilde{z}}{d \omega}=\frac{1}{\pi}\left(\frac{1}{\omega}\right)\left(\frac{\omega-1}{\omega-\gamma^{2}}\right)^{1 / 2} h\left(0^{+}\right),
$$

where $\gamma=h\left(0^{+}\right) / h\left(0^{-}\right)$,

$$
\tilde{z}=\tilde{x}+j \tilde{y}, \quad \hat{x}=\tilde{x}, \quad \hat{y}=h\left(0^{+}\right)-\tilde{y},
$$

and

$$
h\left(0^{+}\right) \equiv 1
$$

(see Fig. 2). Using the substitution

$$
s^{2 \prime}\left(\omega-\gamma^{2}\right) /(\omega-1)
$$

or

$$
\omega=\left(s^{2}-\gamma^{2}\right) /\left(s^{2}-1\right),
$$
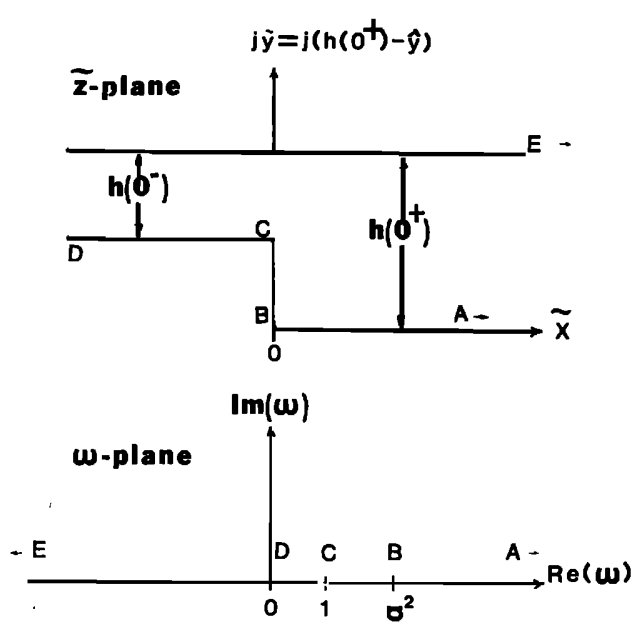

FIG. 2. Conformal transformation from the $\tilde{z}$ plane to the $\omega$ plane where $\gamma=h(+0) / h(-0)$.

Eq. (10) can be rewritten as

$$
\frac{d \tilde{z}}{d s}=-\left(\frac{h\left(0^{+}\right)}{\pi}\right) 2\left(\frac{1}{\left(s^{2}-1\right)}+\frac{1}{\left(\gamma^{2}-s^{2}\right)}\right) .
$$

Integrating with respect to $s$ yields

$\tilde{z}=\left(\frac{h\left(0^{+}\right)}{\pi}\right) \log \left(\frac{(1+s)}{(1-s)}\right)-\left(\frac{h\left(0^{-}\right)}{\pi}\right) \log \left(\frac{(s+\gamma)}{(\gamma-s)}\right)$,

where the nondimensional complex potential is equal to

$$
\Phi=(1 / \pi) \log \omega=j \chi+\hat{\phi},
$$

where $\hat{p}_{n} \equiv i q_{n-1} \operatorname{Re} \Phi$ for $n=1,2$. Using Eqs. (11), (12), and (13), the pressure in the incompressible region can be tabulated.

$$
\begin{aligned}
E_{2} \hat{p}= & \hat{p}_{0}+\epsilon\left[i q_{0}\left(0^{+}\right)+i \epsilon q_{1}\left(0^{+}\right)\right] \operatorname{Re} \Phi[\omega(\hat{x}, \hat{y})] \\
& -\epsilon^{2} k^{2} \hat{p}_{0}\left(\hat{x}^{2} / 2\right)+O\left(\epsilon^{3}\right),
\end{aligned}
$$

where $\Phi$ must be an implicit function of $\hat{x}$ and $\hat{y}$, and $-k^{2} \hat{p}_{0}\left(\hat{x}^{2} / 2\right)$ is a particular solution given in Eq. (8). We must determine the asymptotic behavior of the velocity potential as $\hat{x}$ approaches $\pm \infty$. From these results we can determine the pressure drop and asymptotic values needed to evaluate the composite solution.

Let us first concentrate our effort on determining the asymptotic behavior of the velocity potential in the limit as $\hat{x}$ goes to $-\infty$. Using Eq. (11), we see that as $\hat{x}$ goes to $-\infty, s$ tends to $\gamma$. Therefore, we must solve for the quantity $(s-\gamma)$ in Eq. (12) in order to obtain an expression of the proper form to apply Lagrange's expansion theorem. ${ }^{9}$ Solving for $(s-\gamma)$ in Eq. (12) yields

$$
(s-\gamma)=[(1-s) /(1+s)]^{\gamma}(s+\gamma) \exp \left[\pi \tilde{z} / h\left(0^{-}\right)\right] .
$$

Substituting Eq. (11) in (13) yields

$$
\Phi=(1 / \pi) \log \left\{[(s+\gamma)(s-\gamma)] /\left(s^{2}-1\right)\right\} .
$$

It would seem that Eqs. (15) and (16) are of the appropriate form to apply Lagrange's expansion theorem. However, $\Phi$ is not analytic in the limit as $s$ approaches $\gamma$. By substituting (15) into the above equation yields 
or

$$
\Phi=\left(\frac{1}{\pi}\right) \log \left[\left(\frac{(s+\gamma)^{2}}{\left(s^{2}-1\right)}\right)\left(\frac{(1-s)}{(1+s)}\right)^{\gamma} \exp \left(\frac{\pi \tilde{z}}{h\left(0^{-}\right)}\right)\right]
$$

$$
\Phi=\frac{\tilde{z}}{h\left(0^{-}\right)}+\left(\frac{1}{\pi}\right) \log \left[\left(\frac{(s+\gamma)^{2}}{\left(s^{2}-1\right)}\right)\left(\frac{(1-s)}{(1+s)}\right)^{\gamma}\right] .
$$

Apply Lagrange's expansion theorem to Eqs. (17) and (15) and taking the real part of the result yield

$$
\begin{aligned}
\lim _{\hat{x} \rightarrow-\infty} \operatorname{Re} \Phi= & \frac{\hat{x}}{h\left(0^{-}\right)}+C^{-}+\sum_{n=1}^{\infty} S_{n}^{-} \exp \left(\frac{\hat{x} \pi n}{h\left(0^{-}\right)}\right) \\
& \times \cos \left\{\left[n \pi / h\left(0^{-}\right)\right] \hat{y}\right\}
\end{aligned}
$$

where

$$
\begin{aligned}
& C^{-}=\left(\frac{1}{\pi}\right) \log \left[\left(\frac{(\gamma-1)}{(\gamma+1)}\right)^{\gamma}\left(\frac{4 \gamma^{2}}{\left(\gamma^{2}-1\right)}\right)\right], \\
& S_{1}^{-}=\left(\frac{(\gamma-1)}{(1+\gamma)}\right)^{\gamma}\left(\frac{2}{\pi}\right) \\
& S_{2}^{-}=\left(\frac{(\gamma-1)}{(1+\gamma)}\right)^{2 \gamma}\left(\frac{\left(1-s \gamma^{2}\right)}{\left(1-\gamma^{2}\right)}\right)\left(\frac{1}{\pi}\right),
\end{aligned}
$$

and

$$
\begin{gathered}
S_{n}^{-}=\left(\frac{2}{n !}\right)\left(\frac{d^{n-1}}{d s^{n-1}}\right)\left[\left(\frac{\left(1-\gamma^{2}\right)}{(1-s)}\right)(s+\gamma)^{n-1}\right. \\
\left.=\quad \times\left(\frac{(1-s)}{(1+s)}\right)^{\gamma n}\right]\left.\right|_{s=\gamma} .
\end{gathered}
$$

Following a similar process we can obtain the asymptotic behavior of the velocity potential as $\hat{x}$ approaches $\infty$. As $\hat{x}$ approaches infinity, $s$ approaches 1 . Therefore, solving for $(1-s)$ using Eqs. (11) and (13) yields

$(1-s)=(1+s) \exp \left[\pi z / h\left(0^{+}\right)\right][(s-\gamma) /(s+\gamma)]^{1 / \gamma}$.

The complex velocity potential is

or

$$
\Phi=\left(\frac{1}{\pi}\right) \log \left[-\left(s^{2}-\gamma^{2}\right)\left(\frac{(s-\gamma)}{(s+\gamma)}\right)^{1 / \gamma} \exp \left(\frac{\pi \hat{z}}{h\left(0^{+}\right)}\right)\right]
$$

$$
\Phi=\frac{\hat{z}}{h\left(0^{+}\right)}+\left(\frac{1}{\pi}\right) \log \left[\left(\gamma^{2}-s^{2}\right)\left(\frac{(s+\gamma)}{(s-\gamma)}\right)^{1 / \gamma}\right] .
$$

Applying the Lagrange's expansion theorem to the two aforementioned equations, we can determine the velocity potential in the limit $\hat{x} \rightarrow \infty$.

$$
\begin{aligned}
\lim _{\hat{x} \rightarrow \infty} \operatorname{Re} \Phi= & \frac{\hat{x}}{h\left(0^{+}\right)}+C^{+}+\sum_{n=1}^{\infty} S_{n}^{+} \exp \left(\frac{-n \pi}{h\left(0^{+}\right) \hat{x}}\right) \\
& \times \cos \left[\left(\frac{n \pi}{h\left(0^{+}\right)}\right) \hat{y}\right]
\end{aligned}
$$

where

$$
\begin{aligned}
& C^{+}=\left(\frac{1}{\pi}\right) \log \left[\left(\frac{\left(\gamma^{2}-1\right)}{4}\right)\left(\frac{(\gamma+1)}{(\gamma-1)^{1 / \gamma}}\right)\right], \\
& S_{1}^{+}=-\left(\frac{2}{\pi}\right)\left(\frac{(\gamma-1)}{(1+\gamma)^{1 / \gamma}}\right), \\
& S_{2}^{+}=\left(\frac{1}{\pi}\right)\left(\frac{(\gamma-1)}{(\gamma+1)}\right)^{2 / \gamma}\left(\frac{\left(\gamma^{2}-s\right)}{\left(\gamma^{2}-1\right)}\right),
\end{aligned}
$$

and

$$
\begin{aligned}
S_{n}^{+}= & (-1)^{n}\left(\frac{2}{n !}\right)\left(\frac{d^{n-1}}{d s^{n-1}}\right)\left[\left(\frac{\left(\gamma^{2}-1\right)}{\left(\gamma^{2}-s^{2}\right)}\right)\right. \\
& \left.\times(1+s)^{n-1}\left(\frac{(\gamma-s)}{(s+\gamma)}\right)^{n / \gamma}\right]\left.\right|_{s=1} .
\end{aligned}
$$

The potential drop across the discontinuity is

$$
\begin{aligned}
& \lim _{\hat{x} \rightarrow+\infty} {\left[\operatorname{Re} \Phi-\hat{x} / h\left(0^{-}\right)\right]-\lim _{\hat{x} \rightarrow-\infty}\left[\operatorname{Re} \Phi-\hat{x} / h\left(0^{+}\right)\right] } \\
& \simeq\left(\frac{2}{\pi}\right)\left(\frac{\left(\gamma^{2}-1\right)}{2 \gamma} \log \frac{(1+\gamma)}{(\gamma-1)}+\log \frac{(1+\gamma)^{2}}{4 \gamma}\right)=\Delta \hat{\phi} .
\end{aligned}
$$

If the distance from the end boundaries satisfies the conditions

$$
\begin{aligned}
& l_{1}>\epsilon \log \left(1 / \epsilon^{2}\right) h\left(0^{+}\right) / \pi, \\
& \left|-l_{2}\right|>\epsilon \log \left(1 / \epsilon^{2}\right) h\left(0^{-}\right) / \pi,
\end{aligned}
$$

and if the wall of slope at the $0^{+}$and $0^{-}$is equal to zero, then the aforementioned conditions assure the evanescent waves generated by the nonplanar normal velocity distribution at the discontinuity contribute to the composite solution at or$\operatorname{der} \epsilon^{3}$.

Upon matching ${ }^{10}$ the incompressible solution to the wave solution it can be shown that the junction conditions at the discontinuity are

$$
\begin{aligned}
& p_{0}\left(0^{+}\right)=p_{0}\left(0^{-}\right), \\
& p_{1}\left(0^{+}\right)-p_{1}\left(0^{-}\right)=i \Delta \hat{\phi} q_{0}\left(0^{+}\right), \\
& p_{2}\left(0^{+}\right)-p_{2}\left(0^{-}\right)=i \Delta \hat{\phi} q_{1}\left(0^{+}\right), \\
& q_{0}\left(0^{+}\right)=q_{0}\left(0^{-}\right), \\
& q_{1}\left(0^{+}\right)=q_{1}\left(0^{-}\right),
\end{aligned}
$$

and

$$
q_{2}\left(0^{+}\right)=q_{2}\left(0^{-}\right)
$$

\section{COMPOSITE SOLUTION}

We will now determine the pressure in the coupled space. Matching ${ }^{10}$ the incompressible and wave solutions, the zeroth, first, and second order approximations for pressure will be calculated.

Consider the physical system shown in Fig. 1. We choose $L_{0}$ and $H_{0}$ to equal the length and maximum height of the cavity. Solving Eqs. (4) and (7) to $O(\epsilon)$ and matching the results, the zeroth order composite expansion for pressure can be written as

$$
\begin{aligned}
& E_{0,0} p=\cos \left[k\left(x-l_{1}\right)\right], \quad x \geqslant 0, \\
& E_{0,0} p=\cos k x \cos k l_{1}+\gamma \sin k l_{1} \sin k x, \quad x \leqslant 0,
\end{aligned}
$$

where $h\left(0^{+}\right)=1$, and $\gamma=1 / h\left(0^{-}\right)$. The eigenvalue for the zeroth order composite solution is determined by solving the transcendental equation

$$
\operatorname{cotan} k l_{1}+\gamma \operatorname{cotan} k l_{2}=0 \text {. }
$$

Solving Eqs. (4), (6), and (7) to $O\left(\epsilon^{2}\right)$ and matching the results, the first order expansion for pressure is

$$
\begin{aligned}
E_{1,1} p= & \cos \left[k\left(x-l_{1}\right)\right]+i q_{0} \epsilon[\hat{\phi}(\hat{x}, \hat{y})-\hat{x}] \\
& -i \epsilon q_{0} \hat{\phi}^{+}+O\left(\epsilon^{2}\right), \quad x \geqslant 0,
\end{aligned}
$$

and

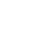




$$
\begin{aligned}
E_{1,1} p= & {\left[\cos k x\left(\cos k l_{1}-\epsilon k \Delta \hat{\phi} \sin k l_{1}\right)\right] } \\
& +\gamma \sin k l_{1} \sin k x+i \epsilon q_{0}[\hat{\phi}(\hat{x}, \hat{y}) \\
& \left.-\hat{x} / h\left(0^{-}\right)\right]-i \epsilon q_{0} \hat{\phi}^{-}+O\left(\epsilon^{2}\right), \quad x \leqslant 0,
\end{aligned}
$$

where

$$
\begin{aligned}
& q_{0}=-i k \sin k l_{1}, \\
& \gamma \equiv 1 / h\left(0^{-}\right), \\
& \hat{\phi}^{-}=-\left(\frac{\gamma}{\pi}\right) \ln \frac{(1+\gamma)}{(\gamma-1)}+\left(\frac{1}{\pi}\right) \ln \frac{4 \gamma^{2}}{\left(\gamma^{2}-1\right)}, \\
& \hat{\phi}^{+}=\left(\frac{1}{\pi \gamma}\right) \ln \frac{(1+\gamma)}{(\gamma-1)}-\left(\frac{1}{\pi}\right) \ln \frac{4^{2}}{(\gamma-1)}
\end{aligned}
$$

and

$$
\begin{aligned}
\Delta \hat{\phi}= & \left(\frac{2}{\pi}\right)\left(\frac{\left(\gamma^{2}-1\right)}{2 \gamma} \ln \frac{(1+\gamma)}{(\gamma-1)}\right. \\
& \left.+\ln \frac{\left(1+\gamma^{2}\right)}{4 \gamma}\right)=\hat{\phi}^{+}-\hat{\phi}^{-},
\end{aligned}
$$

under the condition

$$
\operatorname{cotan} k l_{2}+\gamma \operatorname{cotan} k l_{1}-\epsilon k \Delta \hat{\phi}=0
$$

Since the wall slope equals zero there the $p_{2}$ is equal to zero and $\hat{p}_{2}$ is equal to $-\left(\hat{x}^{2} / 2\right) p_{0}^{\prime \prime}$. Hence,

$$
E_{1,1} p=E_{2,2} p \text {. }
$$

Therefore the composite solution is valid to $O\left(\epsilon^{3}\right)$.

\section{PRESENTATION OF RESULTS AND DISCUSSION OF THE DATA}

An experiment was performed to determine the validity of the composite expansion derived in the previous section. A rectangular test enclosure with inner dimensions measuring $3.92 \mathrm{in} . \times 8.94 \mathrm{in} . \times 1 \mathrm{in}$. was built. The walls of this enclosure were constructed of 1.25 -in. steel bar. The coupled enclosure configuration shown in Fig. 3 was reproduced by inserting a steel block measuring $2.29 \mathrm{in} . \times 1.25 \mathrm{in} . \times 1 \mathrm{in}$. in the corner of the enclosure. Using these dimensions and the nondimensionalization previously discussed, we obtained
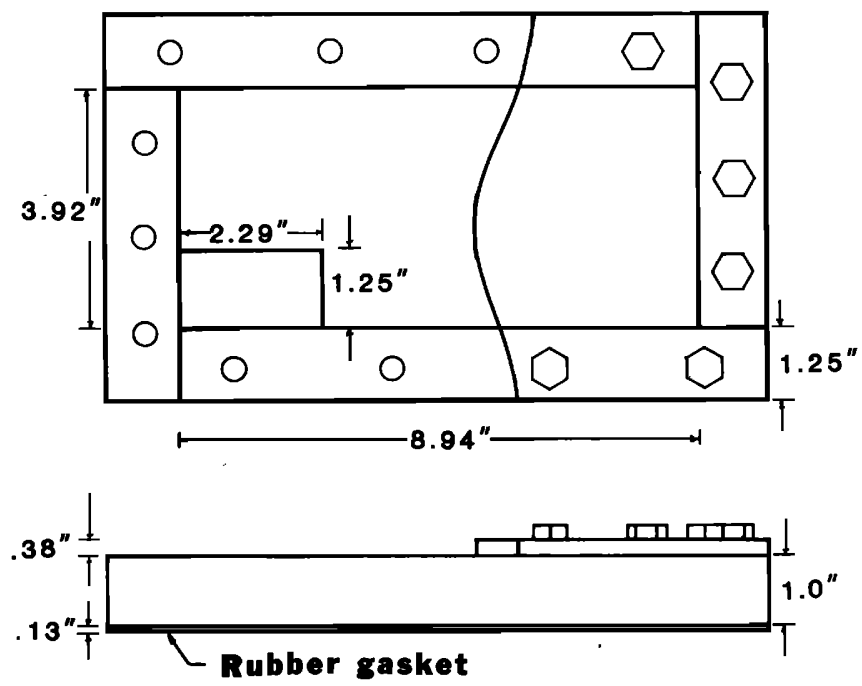

FIG. 3., Model of a coupled space.

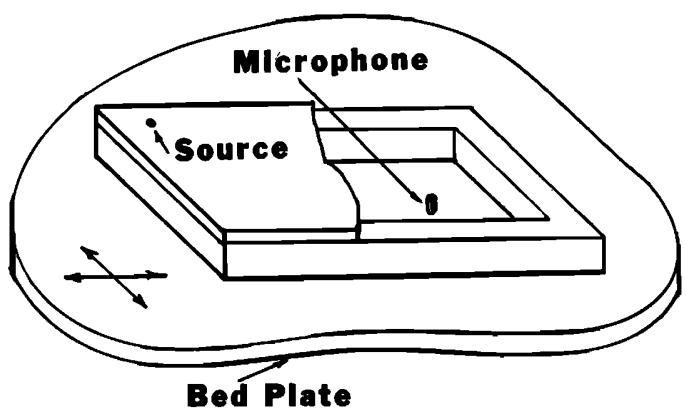

FIG. 4. Simplified drawing of the apparatus used to measure the pressure isobars.

the following values for the characterizing parameters:

$$
\begin{aligned}
& H_{0}=3.92 \text { in., } \\
& L_{0}=8.94 \text { in., } \\
& l_{1}=6.65 \text { in. } / L_{0}=0.744, \\
& l_{2}=2.29 \text { in. } / L_{0}=0.256, \\
& h\left(0^{+}\right)=1, \\
& h\left(0^{-}\right)=0.681,
\end{aligned}
$$

and

$$
\epsilon=0.4386 \text {. }
$$

The top of the enclosure was covered with a 3/8-in. Plexiglas sheet while the bottom was left open. The remaining wall of the enclosure was formed by placing the cavity on a bed plate. By applying a thin layer of soft rubber gasket material to the lower surface of the open side, an airtight seal was formed when cavity was placed on the bed plate (see Fig. 3). A 0.1-in. BBN piezoelectric microphone was installed in the bed plate. The bed plate was then attached to a lathe bed (see Fig. 4). By moving the bed plate relative to the fixed cavity, pressure at any point in the enclosure could be measured.

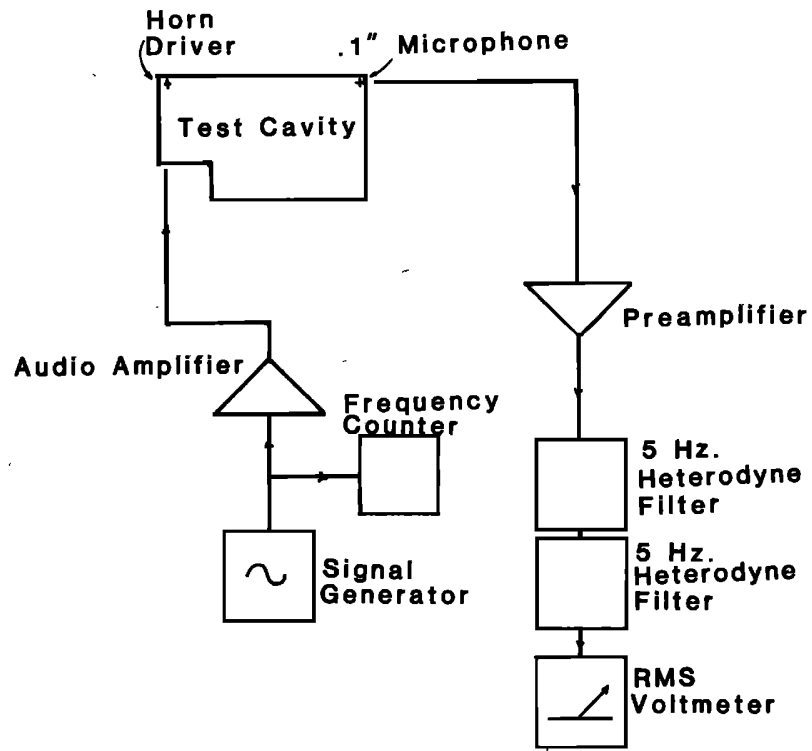

FIG. 5. Block diagram of the experimental setup used in measuring the resonant frequency of the coupled space. 
TABLE 1. First mode measured at $128 \mathrm{~dB}: k(\exp )=3.299, Q=50$.

\begin{tabular}{lcccc}
\hline \hline & $E_{0,0} p$ & Error & $E_{1,1} p$ & Error \\
$\mathrm{k}(\mathrm{cal})$ & 3.331 & $0.96 \%$ & 3.254 & $-1.38 \%$ \\
\hline \hline
\end{tabular}

The system was then excited with a horn driver through a 0.25 -in. hole placed at the top of the cavity.

The resolution in amplitude was $\pm 1 \mathrm{~dB} r e: 20 \mu \mathrm{Pa}$ and spatially to approximately 0.16 -in. as result of the influence of the microphone's finite dimensions.

To measure the first two resonant longitudinal modes of the coupled enclosure, we sinusoidally excited the cavity with the horn driver (see Fig. 5). The sinusoidal signal to the horn driver was provided by an audio amplifier which amplified the sine wave output of a signal generator. The resulting oscillatory pressure was measured with bed plate microphone placed at the adjacent corner of the cavity. To reduce the interference from signals outside the frequency range of interest, the microphone signal was placed through a preamplifier and a cascade of two heterodyne $5-\mathrm{Hz}$ bandwidth filters. These filters were then tuned to the excitation frequency. This arrangement gave us an effective passband of $2.5 \mathrm{~Hz}$. The filtered signal was then measured using a true rms voltmeter and the sound pressure level was then computed.

The resonant frequency of the cavity was determined by adjusting the excitation frequency such that a maximum response was obtained on the voltmeter. A drift of $\pm 5 \mathrm{~Hz}$ in the resonant frequency was encountered when altering over the amplitude range of $120-150 \mathrm{~dB}$. Due to this small nonlinearity all measurements will be presented for fixed pressure amplitude, $128 \mathrm{~dB}$ SPL. The temperature inside the enclosure was also measured. In this way the speed of sound could be accurately determined. Once the peak amplitude and frequency were determined, the bandwidth of the resonance was determined by adjusting the frequency until the measured amplitude was $3 \mathrm{~dB}$ lower than the peak level. The quality factor $Q$ of the resonance was then computed.

The experimental and theoretical results for the eigenvalues of the first two longitudinal modes of the coupled enclosure are given in Tables I and II. For the first mode, the calculated nondimensional wavenumber is $0.96 \%$ too high when the zeroth order composite solution is used and $1.38 \%$ too low when the first order composite solution is used. For the second mode, the calculated nondimensional wavenumber is $6.02 \%$ too high when the zeroth order composite solution is used and $2.3 \%$ too high when the first order composite solution is used.

The quality factor $Q$ for the first and second mode are 50 and 113, respectively. Hence, neglecting damping in our analysis seems to be justified.

TABLE II. Second mode measured at $128 \mathrm{~dB}: k(\exp )=5.888, Q=113$.

\begin{tabular}{lcccc}
\hline \hline & $E_{0,0} p$ & Error & $E_{1,1} p$ & Error \\
$\mathrm{k}(\mathrm{cal})$ & 6.265 & $6.02 \%$ & 6.027 & $2.3 \%$ \\
\hline \hline
\end{tabular}

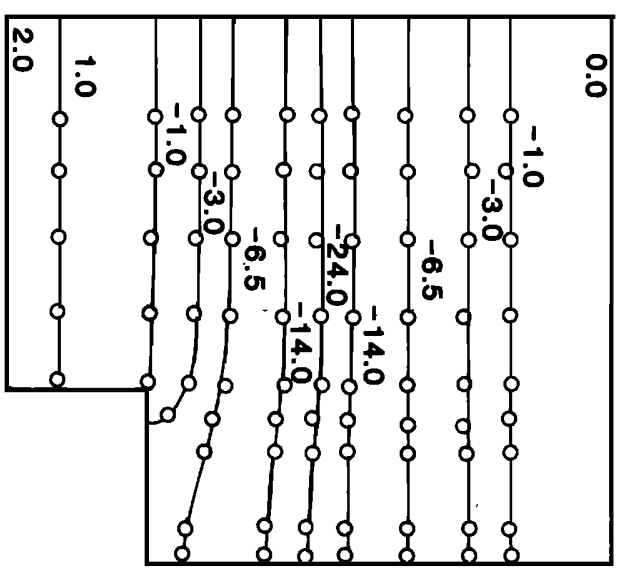

FIG. 6. Experimental result for the acoustic pressure of the first mode. The open circles denote measured data points.

In Figs. 6 and 7 we have plotted the isobars in decibels relative to the sound pressure level in the upper right-hand corner which is $128 \mathrm{~dB}$ SPL. In Fig. 6 the experimental results for the slope of the first mode is shown. We see that in the region about the step discontinuity we have a bending of the isobars. This occurs by virtue of the fact that the vertical velocity is increasing in magnitude in this region. Outside of this region the pressure behaves like a plane standing wave. In Fig. 7 we have plotted the computed isobars for the zeroth order composite solution $E_{0,0} p$. The isobars to this order of approximation are all planar. This is because $E_{0,0} p$ only satisfies continuity in pressure and volume velocity and not the boundary condition of zero normal velocity on the step. We see that computed isobars agree reasonably well with experimental results in areas to the right and left of the discontinuity. Figure 8 is a plot of the computed isobars using the first order composite solution $E_{1,1} p$. We see at this order of solu-
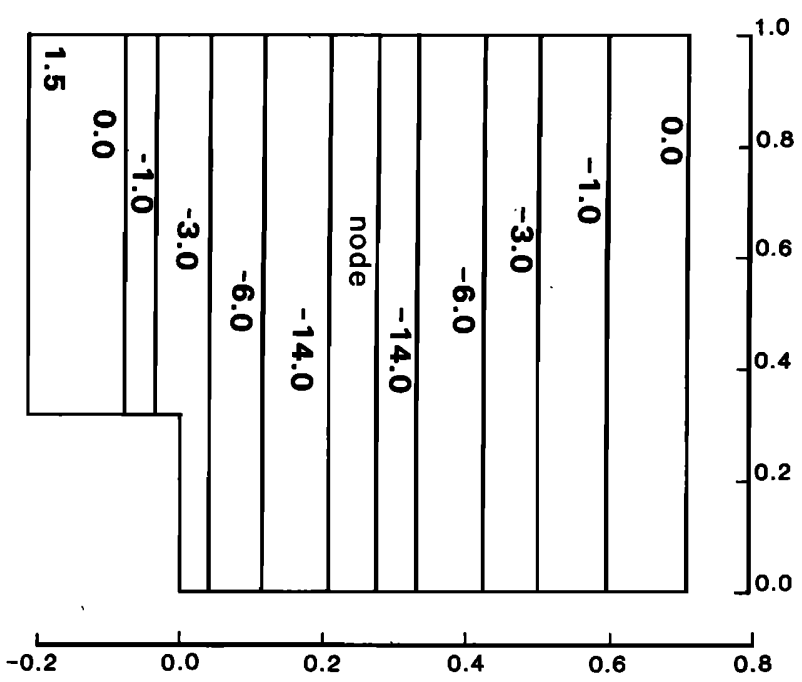

FIG. 7. Plot of the first mode using the composite solution $E_{0,0} p$. 


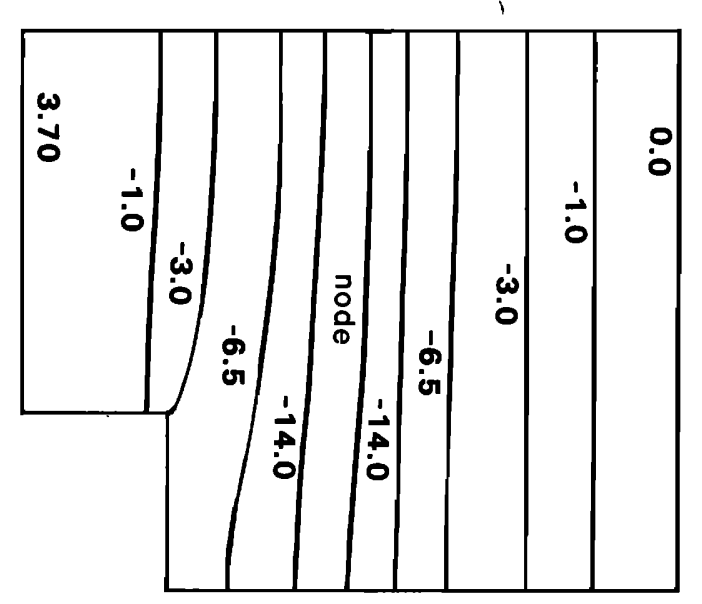

FIG. 8. Plot of the first mode using the composite solution, $E_{1,1} p$.

tion, we have regained the incompressible behavior in the vicinity of the step discontinuity. At this order of approximation both horizontal momentum and volume velocity are conserved at the junction. Note that the predicted eigenvalues given in Table I are higher than the value which would be computed if the block in the cavity were removed. Since the block serves to constrict the flow, the oscillatory particle velocity to the left of the step must increase. The increase in the particle velocity gives rise to a shifting of the nodal line toward the right wall of the cavity. The result is an effective decrease in the wavelength of the standing wave and therefore an increase in the resonant frequency of the system. The mass reactance serves to increase effective length of the cavity. Therefore the adding effect of mass reactance at order $\epsilon$ lowers the resonant frequency.

In Fig. 9 we have plotted the experimental results for the isobars of the second mode of the coupled enclosure. We see that there is a cosine variation in the pressure across the height of the cavity near the right and left walls. It is thought that this variation is the result of influence of the cutoff high-

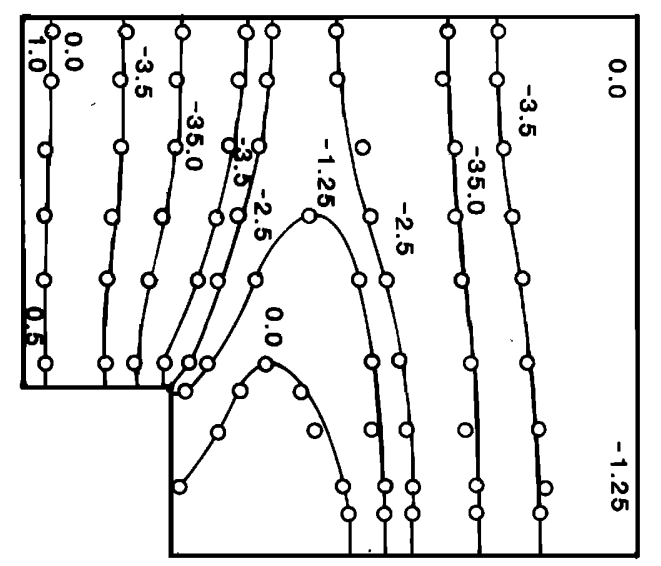

FIG. 9. Experimental result for the acoustic pressure of the second mode. The open circles denote measured data points.

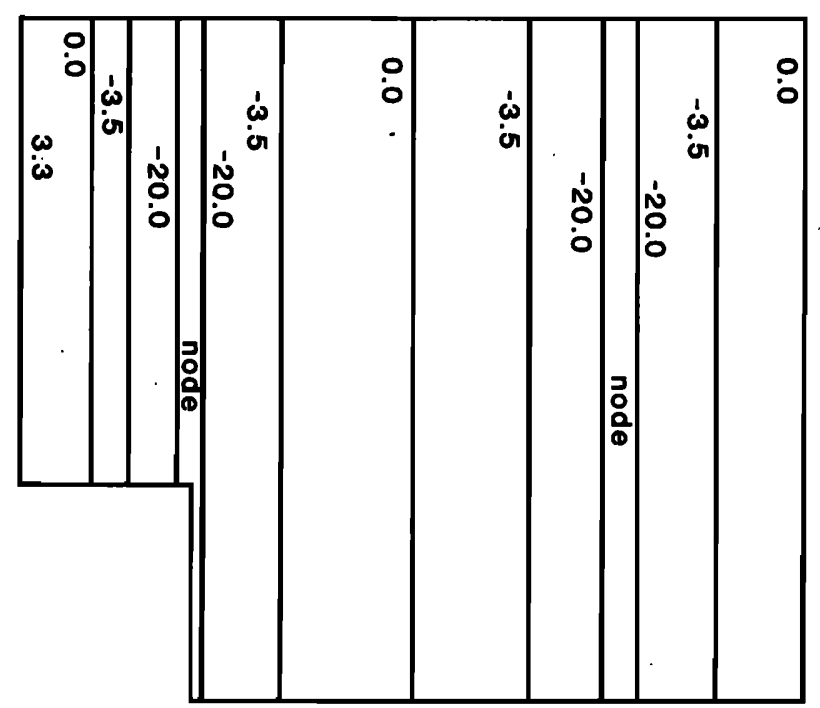

FIG. 10. Plot of the second mode using the composite solution $E_{0,0} p$.

er order cross modes in the cavity. Most importantly the first cross mode appears in the mode shape far from the discontinuity. This is because the resonant wavenumber is approximately 0.8 of the first cross mode wavenumber. In the vicinity of the discontinuity, we have a region of slowly varying pressure. On the lower surface, total variation in pressure is approximately $5 \mathrm{~dB}$ over $1 / 3$ of the cavity's length. Considering the amplitude and spatial resolution of the experiment, determining the absolute location and amplitude of any given isobar in this region is difficult. Hence, we can only say that the experimentally determined isobars shown in this region only represent qualitative estimates of the actual pressure field. In Fig. 10 we have plotted the $E_{0,0} p$ pressure solution. As we can see, $E_{0,0} p$ does not satisfy either the normal velocity boundary condition on the discontinuity nor continuity horizontal momentum. In Fig. 11 we have

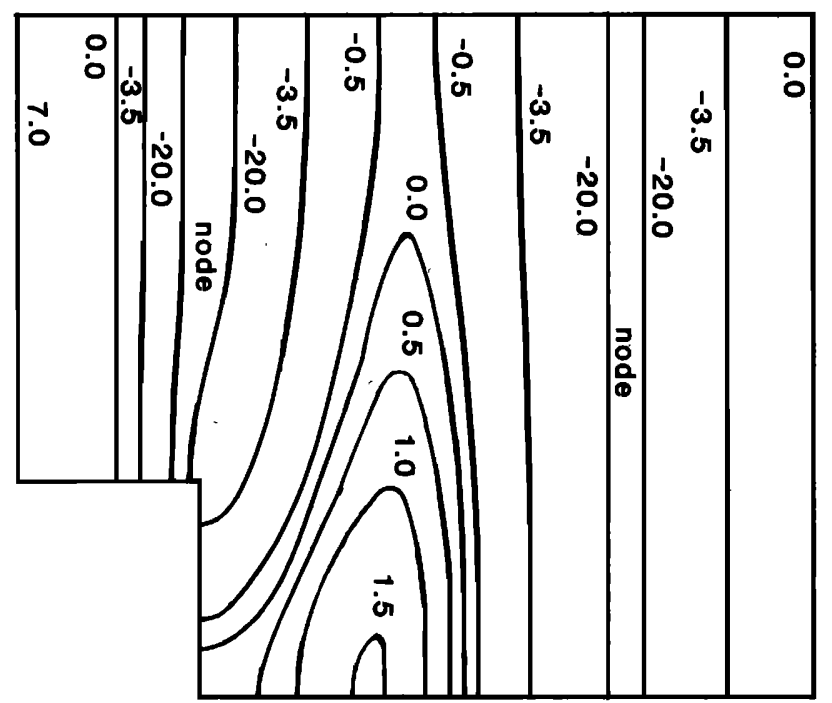

FIG. 11. Plot of the second mode using the composite solution $E_{1,1} p$. 
plotted the first order composite solution $E_{1,1} p$. Incorporating the contribution of higher order terms in the wave field yields a result which is in qualitative agreement with that given in Fig. 9.

Note that the predicted eigenvalues given in Table II are lower than the values which would be obtained if the block in the cavity were removed. The acoustic impedance looking to the left at $x=0$ for values of $k l_{2}$ between $\pi / 2$ and $\pi$ is springlike. The impedance of the left-most cavity, in conjunction with effect the constriction has on the particle velocity tend to move the node toward the left-most wall. The result is an increase in the effective wavelength of the standing wave and therefore a decrease in the resonant frequency of the system. The effect of the mass reactance at order $\epsilon$ serves to further increase the effective length of the cavity. Therefore the resonant frequency is appropriately lowered.

\section{CONCLUDING REMARKS}

An analysis of the wave motion in a coupled space has been presented. It has been shown that even for relatively high values of $\epsilon$ and $k$ the analytical results obtained using MMAE correspond favorably with experiment.

\section{ACKNOWLEDGMENTS}

The author is indebted to Professor Patrick Leehey of the Massachusetts Institute of Technology for valuable suggestions on the earlier version of this manuscript. The author would also like to acknowledge C. Christie and J. McKay for their assistance in the preparation of the manuscript.

${ }^{1}$ A. H. Davis, Philos. Mag. 50, 75 (1925).

${ }^{2}$ C. F. Eyring, J. Acoust. Soc. Am. 3, 181 (1931).

${ }^{3}$ R. H. Bolt, J. Acoust. Soc. Am. 17, 101 (1945).

${ }^{4}$ C. M. Harris and H. Feshbach, J. Acoust. Soc. Am. 22, 572 (1950).

${ }^{5}$ Y. Kagawa and T. Omote, J. Acoust. Soc. Am. 60, 1003 (1976).

${ }^{6}$ M. B. Lesser and J. A. Lewis, J. Acoust. Soc. Am. 51, 1664 (1972).

${ }^{7}$ C. Thompson, J. Acoust. Soc. Am. 74 (1984), to be published.

${ }^{8} \mathrm{C}$. Thompson, J. Acoust. Soc. Am. 74 (1984), to be published.

${ }^{9}$ E. T. Whittaker and G. N. Watson, Modern Analysis (Cambridge U. P. New York, 1927).

${ }^{10} \mathrm{M}$. Van Dyke, Perturbation Methods in Fluid Mechanics (Parabolic, Stanford, CA, 1964). 